\title{
Impurity-induced local density of states in a $d$-wave superconductor carrying a supercurrent
}

\author{
Degang Zhang, ${ }^{1}$ C. S. Ting, ${ }^{1}$ and C.-R. $\mathrm{Hu}^{2}$ \\ ${ }^{1}$ Texas Center for Superconductivity and Department of Physics, University of Houston, Houston, Texas 77204, USA \\ ${ }^{2}$ Department of Physics, Texas A \& M University, College Station, Texas 77843, USA
}

(Received 19 October 2004; published 28 February 2005)

\begin{abstract}
The local density of states (LDOS) and its Fourier component induced by a unitary impurity in a supercurrent-carrying $d$-wave superconductor are investigated. Both of these quantities possess a reflection symmetry about the line passing through the impurity site and along the supercurrent if it is applied along the antinodal or nodal direction. With increasing supercurrent, both the coherence and resonant peaks in LDOS are suppressed and slightly broadened. Under a supercurrent along the antinodal direction, the coherence peaks split into double peaks. The modulation wave vectors associated with the elastic scatterings of quasiparticles by the defect from one constant-energy piece of the Fermi surface to another are displayed as bright or dark spots in the Fourier space of the LDOS image, and they may be suppressed or enhanced and shifted, depending on the applied current and the bias voltage.
\end{abstract}

DOI: 10.1103/PhysRevB.71.064521

PACS number(s): 74.25.-q, 74.20.-z, 74.62.Dh

The understanding of the local physics in cuprate or hightemperature superconductors (HTS) is one of the most challenging problems in condensed matter physics today. Different from the conventional $s$-wave superconductors, the HTS have very complex phase diagrams depending on doping and chemical composition. It is also well established that the superconducting order parameter in the cuprates has predominantly $d$-wave symmetry. ${ }^{1}$ The zero-bias conductance peak (ZBCP) in the tunneling spectroscopy of normal metalcuprate superconductor junction with non- $(\mathrm{n} 0 \mathrm{~m})$-directional contact provides one of the direct evidences for this symmetry. ${ }^{2}$ Due to the $d$-wave nature of the order parameter, impurities inserted into cuprates can serve as an important tool to explore the physics of HTS. Theoretical calculations of the local density of states (LDOS) predicted that a strong potential scatterer could induce a resonance peak near the Fermi level at sites near the impurity. ${ }^{3,4}$ This resonant peak near zero-bias voltage was observed at and near the sites of $\mathrm{Zn}$ impurities in $\mathrm{Bi}_{2} \mathrm{Sr}_{2} \mathrm{CaCu}_{2} \mathrm{O}_{8+\delta}$ by scanning tunneling microscopy (STM). ${ }^{5}$ In addition, an interference pattern with fourfold symmetry was also detected in the STM image. ${ }^{5}$

When a superconductor carries a supercurrent $\left(J_{S}\right)$, Cooper pairs with finite momentum appear in the system. This would drastically affect the electronic structure of the superconductor, including the elementary excitation spectrum, the order parameter symmetry, and the tunneling spectroscopy. ${ }^{6-8}$ With increasing supercurrent velocity, the superconducting order parameter can be depressed. Meanwhile, the supercurrent density first increases monotonously and then arrives at a maximum value, which is called the critical current density. Beyond that, superconductivity becomes unstable and collapses to the normal state. So the supercurrent in the stable regime can be also used as a probe to further understand the quasiparticle excitations in HTS. Moreover, a better understanding of the property of a superconductor under an applied $J_{s}$ may have the potential for device applications.

In Ref. 7, we have studied the tunneling conductance characteristics between a normal metal and a $d$-wave super- conductor (dSC) carrying a supercurrent parallel to the interface of the junction. It was shown that for sufficiently large applied current, the (midgap surface state)-induced ZBCP splits into two peaks in the tunneling regime. So far there exist no experimental measurements which could be used to compare with our theoretical predictions. The closest tunneling experiment to the idea in Ref. 7 was done on $\mathrm{YBa}_{2} \mathrm{Cu}_{3} \mathrm{O}_{7-\delta}$ under a spin-injected current. ${ }^{9}$ It would be interesting to see a similar experiment performed on an HTS sample carrying a supercurrent in the near future. As a natural extension of our previous work, ${ }^{7}$ here we examine the LDOS induced by a strong defect which replaces a $\mathrm{Cu}^{2+}$ ion in the top $\mathrm{CuO}$ layer of a current carrying HTS. This strong defect could either be a unitary impurity like $\mathrm{Zn}^{2+}$ or simply a $\mathrm{Cu}$ vacancy, and is well known to induce a near-zero bias resonant peak (NZBRP) next to the site of the defect in a dSC without $J_{s}{ }^{3,4}$ In the following, we investigate the LDOS images and their Fourier components for several values of the bias energies $E$ and $J_{s}$. In addition the LDOS at sites next to and far away from the impurity as functions of $E$ are calculated and their behaviors under various $J_{s}$ will be presented and discussed.

The BCS Hamiltonian describing the impurity effects in a dSC carrying a supercurrent can be written as

$$
\begin{aligned}
H= & \sum_{\mathbf{k} \sigma}\left(\epsilon_{\mathbf{k}}-\mu\right) c_{\mathbf{k} \sigma}^{+} c_{\mathbf{k} \sigma}+\sum_{\mathbf{k}}\left[\Delta_{\mathbf{q}_{s}}(\mathbf{k}) c_{\mathbf{k}+\mathbf{q}_{s} \uparrow}^{+} c_{-\mathbf{k}+\mathbf{q}_{s} \downarrow}^{+}+\text {H.c. }\right] \\
& +V_{s} \sum_{\sigma} c_{0 \sigma}^{+} c_{0 \sigma}
\end{aligned}
$$

where $\epsilon_{\mathbf{k}}$ is the band structure of the $d$-wave superconductor, $\mu$ is the chemical potential to be determined by doping, $V_{s}$ is the on-site potential of the nonmagnetic impurity located at the center of lattice, $\mathbf{q}_{s}=\left(m_{*} / 2\right) \mathbf{v}_{s}$ with $\mathbf{v}_{s}$ the supercurrent velocity, $m_{*}$ is the mass of a Cooper pair, $\Delta_{\mathbf{q}_{s}}(\mathbf{k})=\Delta_{\mathbf{q}_{s}} \cos (2 \theta)$ is the superconducting order parameter in the presence of $J_{s}, \theta$ is the angle between the wave vector $\mathbf{k}$ and the antinodal direction of the $d$-wave superconductor, and $\Delta_{\mathbf{q}_{s}}$ is determined by the gap equation, ${ }^{7}$ 


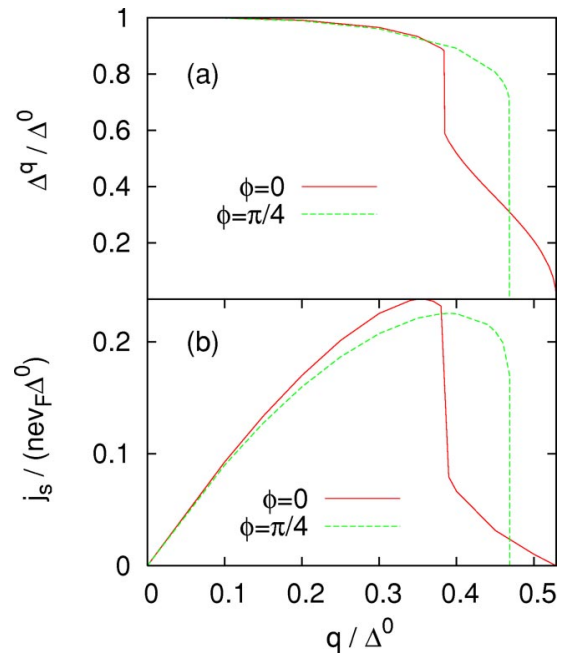

FIG. 1. (Color online) (a) Dependences of the normalized superconducting order parameter $\Delta^{q}$ on the normalized supercurrentvelocity parameter $q$ for a $d$-wave superconductor and (b) the corresponding dependences of supercurrent density $j_{s}$ on $q$.

$$
\pi \ln \frac{\Delta^{0}}{\Delta^{q}}=\int_{\geqslant} d \theta \cos ^{2}(2 \theta) \ln \left[g(\phi)+\sqrt{g^{2}(\phi)-1}\right],
$$

where

$$
g(\phi) \equiv \frac{2 q}{\Delta^{q}}\left|\frac{\cos (\theta-\phi)}{\cos (2 \theta)}\right|, \quad q \equiv \frac{q_{s}}{k_{F}}, \quad \Delta^{q} \equiv \frac{\Delta_{\mathbf{q}_{s}}}{E_{F}} .
$$

$k_{F}$ and $E_{F}$ are the Fermi momentum and energy, respectively, $\phi$ is the angle between $\mathbf{q}_{s}$ and the antinodal direction, and the integration in Eq. (2) is from 0 to $2 \pi$ with the constraint $g^{2}(\phi)-1 \geqslant 0$. The solutions of Eq. (2) with $\phi=0$ and $\pi / 4$ are presented in Fig. 1(a). In Ref. 7, we also derived the thermodynamic critical currents $j_{s c}(0)=0.238 e^{e n v_{F}} \Delta_{0}$ at $q=q_{c}(0)=0.35 \Delta_{0}$ and $j_{s c}(\pi / 4)=0.225 e n v_{F} \Delta_{0}$ at $q=q_{c}(\pi / 4)$ $=0.39 \Delta_{0}$ for supercurrent $J_{s}$ along the antinodal and nodal directions, respectively [see Fig. 1(b)]. Similar results on the order parameter and the critical currents have also been obtained in Ref. 8.

The Hamiltonian (1) is exactly soluble by the Bogoliubov transformation and the Green's function technique. ${ }^{10}$ After a tedious but straightforward calculation, we obtain the expression for LDOS near a strong impurity in the unitary limit (i.e., $V_{s} \rightarrow \infty$ )

$$
\begin{gathered}
\rho(\mathbf{r}, \omega)=\rho_{0}(\mathbf{r}, \omega)+\delta \rho(\mathbf{r}, \omega), \\
\rho_{0}(\mathbf{r}, \omega)=-\left.\frac{2}{\pi \mathcal{N}} \operatorname{Im} \sum_{\mathbf{k}, \nu} \xi_{\mathbf{k} \nu}^{2}\left(\mathbf{q}_{s}\right) G_{\mathbf{k} \nu}^{0}\left(\mathbf{q}_{s}, i \omega_{n}\right)\right|_{i \omega_{n} \rightarrow \omega+i 0^{+}}, \\
\delta \rho(\mathbf{r}, \omega)=-\frac{2}{\pi \mathcal{N}^{2}} \operatorname{Im} \sum_{\mathbf{k}, \mathbf{k}^{\prime}} \sum_{\nu, \nu^{\prime}=0,1} D\left(\mathbf{q}_{s}, i \omega_{n}\right) \cos \left[\left(\mathbf{k}-\mathbf{k}^{\prime}\right) \cdot \mathbf{r}\right] \\
\times\left\{\xi_{\mathbf{k} \nu}^{2}\left(\mathbf{q}_{s}\right) \xi_{\mathbf{k}^{\prime} \nu^{\prime}}^{2}\left(\mathbf{q}_{s}\right) b\left(\mathbf{q}_{s}, i \omega_{n}\right)\right. \\
-2(-1)^{\nu} \xi_{\mathbf{k} \nu}\left(\mathbf{q}_{s}\right) \xi_{\mathbf{k} \nu+1}\left(\mathbf{q}_{s}\right) \xi_{\mathbf{k}^{\prime} \nu^{\prime}}^{2}\left(\mathbf{q}_{s}\right) c\left(\mathbf{q}_{s}, i \omega_{n}\right)
\end{gathered}
$$

$$
\begin{aligned}
& +(-1)^{\nu+\nu^{\prime}} \xi_{\mathbf{k} \nu}\left(\mathbf{q}_{s}\right) \xi_{\mathbf{k} \nu+1}\left(\mathbf{q}_{s}\right) \xi_{\mathbf{k}^{\prime} \nu^{\prime}}\left(\mathbf{q}_{s}\right) \xi_{\mathbf{k}^{\prime} \nu^{\prime}+1}\left(\mathbf{q}_{s}\right) \\
& \left.\times a\left(\mathbf{q}_{s}, i \omega_{n}\right)\right\}\left.G_{\mathbf{k} \nu}^{0}\left(\mathbf{q}_{s}, i \omega_{n}\right) G_{\mathbf{k}^{\prime} \nu^{\prime}}^{0}\left(\mathbf{q}_{s}, i \omega_{n}\right)\right|_{i \omega_{n} \rightarrow \omega+i 0^{+}}
\end{aligned}
$$

where $\mathcal{N}$ is the site number of lattice, and

$$
\begin{aligned}
& E_{\mathbf{q}_{s}}(\mathbf{k})= \sqrt{\left[\frac{1}{2}\left(\epsilon_{\mathbf{k}+\mathbf{q}_{s}}+\epsilon_{-\mathbf{k}+\mathbf{q}_{s}}\right)-\mu\right]^{2}+\Delta_{\mathbf{q}_{s}}^{2}(\mathbf{k})}, \\
& \xi_{\mathbf{k} \nu}^{2}\left(\mathbf{q}_{s}\right)= \frac{1}{2}\left[1+(-1)^{\nu} \frac{\frac{1}{2}\left(\epsilon_{\mathbf{k}+\mathbf{q}_{s}}+\epsilon_{-\mathbf{k}+\mathbf{q}_{s}}\right)-\mu}{E_{\mathbf{q}_{s}}(\mathbf{k})}\right], \\
& \xi_{\mathbf{k} 0}\left(\mathbf{q}_{s}\right) \xi_{\mathbf{k} 1}\left(\mathbf{q}_{s}\right)=\frac{\Delta_{\mathbf{q}_{s}}(\mathbf{k})}{2 E_{\mathbf{q}_{s}}(\mathbf{k})},
\end{aligned}
$$

$$
\begin{gathered}
G_{\mathbf{k} \nu}^{0}\left(\mathbf{q}_{s}, i \omega_{n}\right)=\frac{1}{i \omega_{n}-\frac{1}{2}\left(\epsilon_{\mathbf{k}+\mathbf{q}_{s}}-\epsilon_{-\mathbf{k}+\mathbf{q}_{s}}\right)-(-1)^{\nu} E_{\mathbf{q}_{s}}(\mathbf{k})}, \\
a\left(\mathbf{q}_{s}, i \omega_{n}\right)=\frac{1}{\mathcal{N}} \sum_{\mathbf{k}, \nu} \xi_{\mathbf{k} \nu}^{2}\left(\mathbf{q}_{s}\right) G_{\mathbf{k} \nu}^{0}\left(\mathbf{q}_{s}, i \omega_{n}\right), \\
b\left(\mathbf{q}_{s}, i \omega_{n}\right)=\frac{1}{\mathcal{N}} \sum_{\mathbf{k}, \nu} \xi_{\mathbf{k} \nu+1}^{2}\left(\mathbf{q}_{s}\right) G_{\mathbf{k} \nu}^{0}\left(\mathbf{q}_{s}, i \omega_{n}\right), \\
c\left(\mathbf{q}_{s}, i \omega_{n}\right)=\frac{1}{\mathcal{N}} \sum_{\mathbf{k}, \nu}(-1)^{\nu} \xi_{\mathbf{k} \nu}\left(\mathbf{q}_{s}\right) \xi_{\mathbf{k} \nu+1}\left(\mathbf{q}_{s}\right) G_{\mathbf{k} \nu}^{0}\left(\mathbf{q}_{s}, i \omega_{n}\right), \\
D\left(\mathbf{q}_{s}, i \omega_{n}\right)=\frac{1}{c^{2}\left(\mathbf{q}_{s}, i \omega_{n}\right)-a\left(\mathbf{q}_{s}, i \omega_{n}\right) b\left(\mathbf{q}_{s}, i \omega_{n}\right)} .
\end{gathered}
$$

Obviously, when a supercurrent is applied, the quasiparticle energy has a momentum-dependent shift $1 / 2\left(\epsilon_{\mathbf{k}+\mathbf{q}_{s}}\right.$ $\left.-\epsilon_{-\mathbf{k}+\mathbf{q}_{s}}\right)$ [see the bare Green's function $G_{\mathbf{k} \nu}^{0}\left(\mathbf{q}_{s}, i \omega_{n}\right)$ in Eq. (5)], which leads to different gaps for different momentum directions of a quasiparticle. This strongly modifies the LDOS and its Fourier components patterns. We shall see that the variation of these patterns is sensitive to the supercurrent applied and the bias voltage; however, they always have a reflection symmetry at an arbitrary energy if a supercurrent is applied along the antinodal or nodal directions. From Eqs. (4) and (5), we calculate the LDOS at several different energies and supercurrent velocities with the defect located at the center of a $\mathcal{N}=400 \times 400$ lattice. Here we adopt the band structure of $\mathrm{Bi}_{2} \mathrm{Sr}_{2} \mathrm{CaCu}_{2} \mathrm{O}_{8+\delta}$ given by Norman et al., $\epsilon_{\mathbf{k}}=-0.5951\left(\cos k_{x}+\cos k_{y}\right) / 2+0.1636 \cos k_{x} \cos k_{y}-0.0519$ $\left(\cos 2 k_{x}+\cos 2 k_{y}\right) / 2-0.1117\left(\cos 2 k_{x} \cos k_{y}+\cos k_{x} \cos 2 k_{y}\right)$ $12+0.0510 \cos 2 k_{x} \cos 2 k_{y}-\mu(\mathrm{eV}),{ }^{11}$ which corresponds to that of free electrons with $k_{F}=1.639$ and $E_{F}=0.4203 \mathrm{eV}$, for the chemical potential $\mu=-0.1238 \mathrm{eV}$ for optimal doping (15\%). Choosing $\Delta_{0}=44 \mathrm{meV}$, and $\Delta_{\mathbf{q}}$ can be obtained from Fig. 1(a).

Fig. 2 shows the $20 \times 20$ images of the $\operatorname{LDOS} \rho(\mathbf{r}, \omega)$ at different energies and supercurrents with the impurity at its center. In order to understand the image patterns in Fig. 2, 

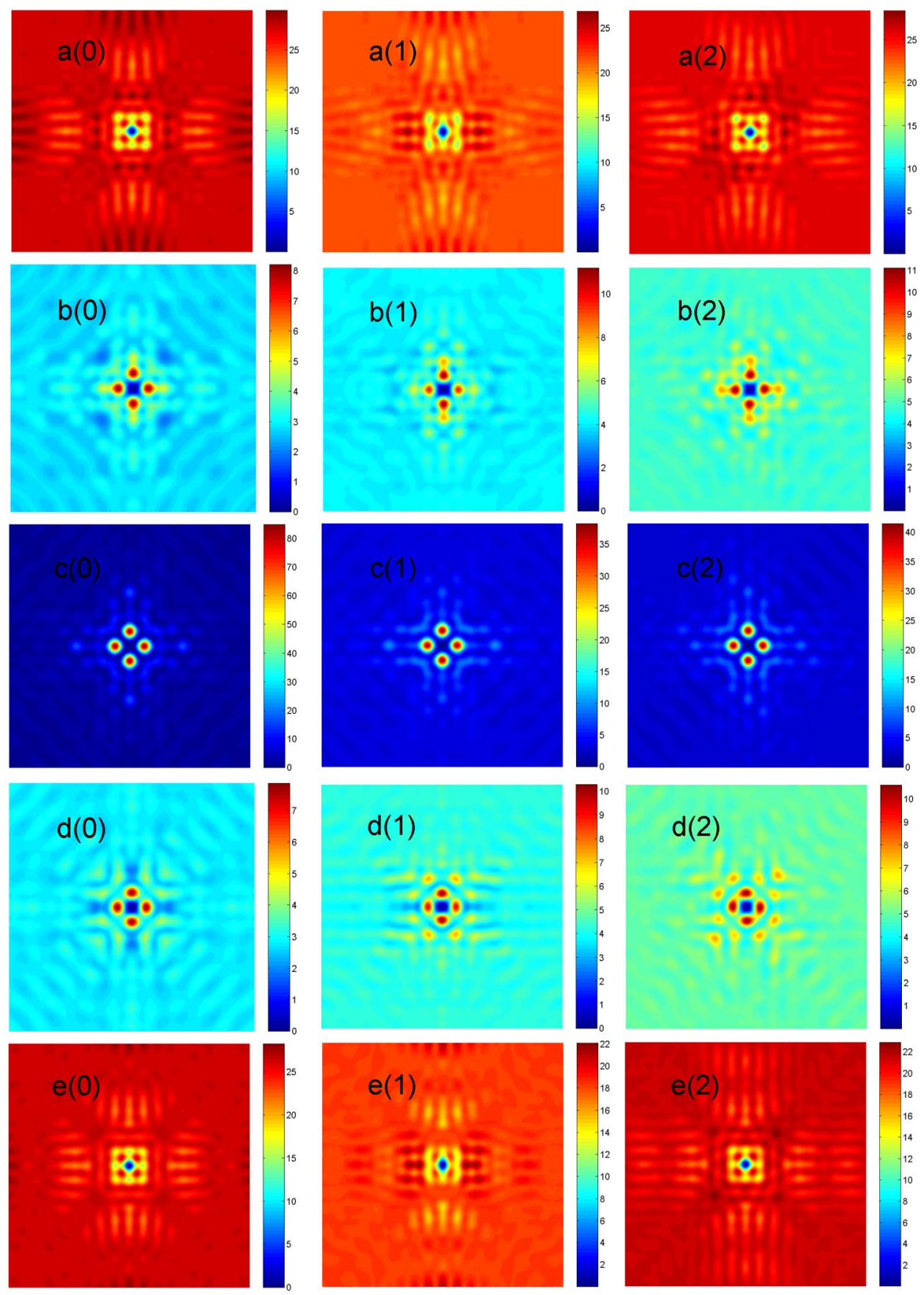

FIG. 2. (Color online) The $20 \times 20$ images of the LDOS $\rho(\boldsymbol{r}, \omega)$ at $\omega=-44,-16,0,16$, and $44 \mathrm{meV}$ (from top to bottom) and $q=0, q_{c}(0)$, and $q_{c}(\pi / 4)$ (from left to right) for a unitary impurity at its center. we plot the schematic Fermi surface of an optimally doped HTS in the first Brillouin zone as shown in Fig. 3. In an STM experiment and when a quasiparticle is created near the Fermi surface at point $\mathrm{O}$, this quasiparticle may be scattered elastically by the defect to other equivalent points (such as A, B, C, D, E, F, and G) near the Fermi surface. ${ }^{12-16}$ The wave vectors connecting $\mathrm{O}$ and the other points are called the modulation wave vectors and they are labeled as $q_{A}, q_{B}$, etc., up to $q_{F}$. If the point $\mathrm{O}$ is at the middle of the Fermi curve in Fig. 3, then the quasiparticle is at the nodal point. If $\mathrm{O}$ is moved to the zone boundary, then the quasiparticle is at the antinodal point. Because of the $d$-wave nature of the superconductivity, little energy is required to create a quasiparticle at the nodal point. To create a quasiparticle at the antinodal point, however, a large bias energy in the order of the superconductivity gap is needed in an STM experiment.

From Fig. 2, the LDOS at the impurity site vanishes regardless of the bias energies and the strength of $J_{s}$, and it has the strongest intensity at the sites next to the defect when the quasiparticle energy (or the bias voltage times $e$ ) $\omega=0 \mathrm{meV}$ [see Fig. 2(c) (0)]. Near the impurity, the LDOS has a pattern of fourfold symmetry with energy-dependent modulations in the absence of a supercurrent [Figs. 2(a) (0) to $2(\mathrm{e})(0)]$.

When $|\omega|=0$ and $16 \mathrm{meV}$, the resonant peaks still show up at $(0, \pm 1)$ and $( \pm 1,0)$ and the modulation with the periodicity $\sim 2 a$ is along $45^{\circ}$ from the $\mathrm{Cu}-\mathrm{O}$ bonds. For $\omega=0 \mathrm{meV}$, the point $\mathrm{O}$ is at the nodal point, and the modulation in Fig. 


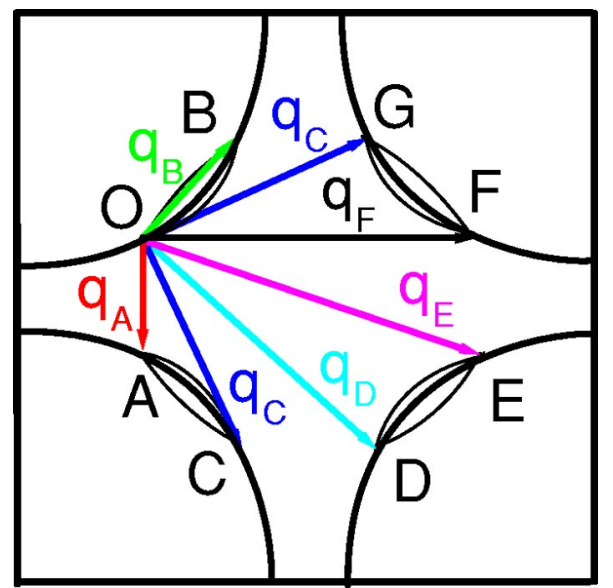

FIG. 3. (Color online) Schematic Fermi surface of high- $T_{C}$ cuprate superconductor. The modulation wave vectors connecting different points of the Fermi surface with the same energy gap are shown in the absence of a supercurrent.

2(c) (0) comes from the wave vector $q_{D}=q_{E}$ in Fig. 3. For $|\omega|=16 \mathrm{meV}$, the pattern seems to be a result of combined contributions from $q_{B}$ and other modulation wave vectors along the directions $( \pm \pi, \pm \pi)$.

When $|\omega|=44 \mathrm{meV}$, the point $\mathrm{O}$ moves to the zone boundary or the antinodal point. The modulation wave vector $q_{F}=2 \pi$. This would give rise an to $x$-oriented and $y$-oriented (or $\mathrm{Cu}-\mathrm{O}$ bond oriented) stripe-like structure with the periodicity $\sim a$ in the LDOS around the impurity, and this can be seen clearly in Fig. 2(a) (0). The LDOS here also exhibits a checkerboard pattern close to the impurity site due to the combined effects of the $x$-oriented and $y$-oriented stripes.

When a supercurrent $J_{s}$ is applied along the antinodal direction (i.e., $\phi=0$ along the $x$-direction), the intensities of the resonance peaks on points $(0, \pm 1)$ are higher than those on the points $( \pm 1,0)$ at $|\omega|=0$ and $16 \mathrm{meV}$. Near the critical current $j_{s c}(0)$, the LDOS develops a modulation perpendicular to the direction of supercurrent at $|\omega|=16 \mathrm{meV}$. When $|\omega|=44 \mathrm{meV}$, the intensity of the modulation parallel to $J_{s}$ becomes smaller than that perpendicular to $J_{s}$. When $J_{s}$ is applied along the nodal direction (i.e., $\phi=\pi / 4$ from the $x$ axis), the LDOS patterns only have minor changes except that some brighter spots near the defect site appear at $|\omega|=16 \mathrm{meV}$. We note that with increasing $J_{s}$, the maxima of the LDOS at $|\omega|=0$ and $44 \mathrm{meV}$ are suppressed, while those at $|\omega|=16 \mathrm{meV}$ are enhanced.

In order to further understand the supercurrent effects, we also calculate the images for the Fourier component of the LDOS (FCLDOS) (see Fig. 4). It can be easily seen that the influence of the applied supercurrent on the FCLDOS is more dramatic than on the LDOS image. Here some modulation wave vectors corresponding to the elastic scattering of quasiparticles from one point of the Fermi surface to another point as shown in Fig. 3 can be clearly identified in Fig. 4.

When $\omega=0 \mathrm{meV}$ and $q_{s}=0$, the modulation wave vectors $q_{A}=q_{C}=q_{F}$ and $q_{D}=q_{E}$, due to the nodal quasiparticle scattering, are clearly seen in the FCLDOS patterns [Fig. 4(c) (0)]. We note that the dip at $q_{D, E}$ has a strong intensity, which causes the LDOS to have a modulation along $45^{\circ}$ to
$\mathrm{Cu}-\mathrm{O}$ bonds [Fig. 4(c) (0)]. At the critical currents $J_{s c}(0)$ and $J_{s c}(\pi / 4)$, the dips are suppressed, but their positions seem not to shift [Figs. 4(c) (1) and 4(c) (2)].

At higher energy, however, the case becomes more complicated. At $|\omega|=16 \mathrm{meV}$, the dips at $q_{A}$ and $q_{F}$ are clearly visible in the absence of supercurrent [Figs. 4(b) (0) and 4(d) (0)]. At four corners of the first Brillouin zone, there are four arcs due to the scatterings of the quasiparticles by the defect from one equal-energy banana contour (e.g., arcs $\mathrm{OB}$ in Fig. 3) to the opposite contour (e.g., arc DE in Fig. 3). It is these arcs that mainly produce the charge modulation along $45^{\circ}$ from the $\mathrm{Cu}-\mathrm{O}$ bonds [Figs. 2(b) (0) and 2(d) (0)]. At $\omega=-16 \mathrm{meV}$, we note that the peaks associated with $q_{B}$ are absent. Instead, four new dips at $q_{2}$ show up. When a $J_{s}$ is applied, these peaks and arcs for $|\omega|=16 \mathrm{meV}$ are suppressed or enhanced, and even vanish near the critical current, but their positions have little shift [see Figs. 4(b) (1), 4(b) (2), 4(d) (1), and 4(d) (2)].

When $|\omega|=44 \mathrm{meV}$, Figs. 4(a) (0) and 4(e) (0) show the images of the FCLDOS associated with the scattering of antinodal quasiparticles. The peaks corresponding to $q_{A}$ and $q_{1}$ are clearly seen here. The modulation wave vectors $q_{1}$ are due to the superposition of those peak arcs induced by the scatterings of quasiparticles from one antinodal part of the banana contour to the neighboring part. Obviously, the equalenergy banana contour becomes wide for $J_{s}$ along the antinodal direction while it shrinks to a point for $J_{s}$ along the nodal direction [Figs. 4(a) (1), 4(a) (2), 4(e) (1), and 4(e) (2)]. From Figs. 2 and 4, it can be seen clearly that the effect of $J_{s}$ on the FCLDOS is much more pronounced than that on LDOS.

In order to examine the change of modulation wave vectors with $J_{s}$, we present the FCLDOS along the antinodal and nodal directions at $\omega=0 \mathrm{meV}$ and for several supercurrent strengths in Fig. 5. With increasing supercurrent velocity, dips associated with $q_{A, C, F}$ are suppressed and finally disappear [see Figs. 5(a) and 5(c)]. However, the dips corresponding to $q_{D, E}$ are only suppressed for $J_{s}$ along the antinodal direction, or $\phi=0$, while they are first enhanced, then weakened, and have a tiny shift for the $J_{s}$ along the nodal direction, or $\phi=\pi / 4$ [Figs. 5(b) and 5(d)]. Similar results hold at higher energy. We note that the dips at $|q|=1.6 \pi$ in Figs. 5(a) and 5(c) cannot be induced by quasiparticle scatterings, which are also suppressed with increasing $J_{s}$. We think that these dips at $q_{A, C, F}$ and $q_{D, E}$ are due to the manifestation of quasiparticle destructive interference due to the sign change of the $d$-wave gap function on the Fermi surface.

We have obtained the LDOS and FCLDOS induced by a strong defect such as a Zn impurity. Now we turn our attention to the STM experiments. In the STM experiments, ${ }^{5}$ a zero-bias resonant peak was observed at the $\mathrm{Zn}$ sites; however, theoretical calculations give a vanishing LDOS at the impurity sites, contrary to the experimental observation (see Fig. 2). Because a $\mathrm{Bi}$ atom in the top (BiO) layer and, more importantly, an $\mathrm{O}$ atom in the second $(\mathrm{SrO})$ layer block the tunneling current coming from the STM tip to directly probe the impurity site,$^{17}$ the experimentally observed LDOS at the impurity or $\mathrm{Cu}$ site should be approximately equal to the sum of those on the four nearest neighbor sites around it, ${ }^{18}$ i.e., 

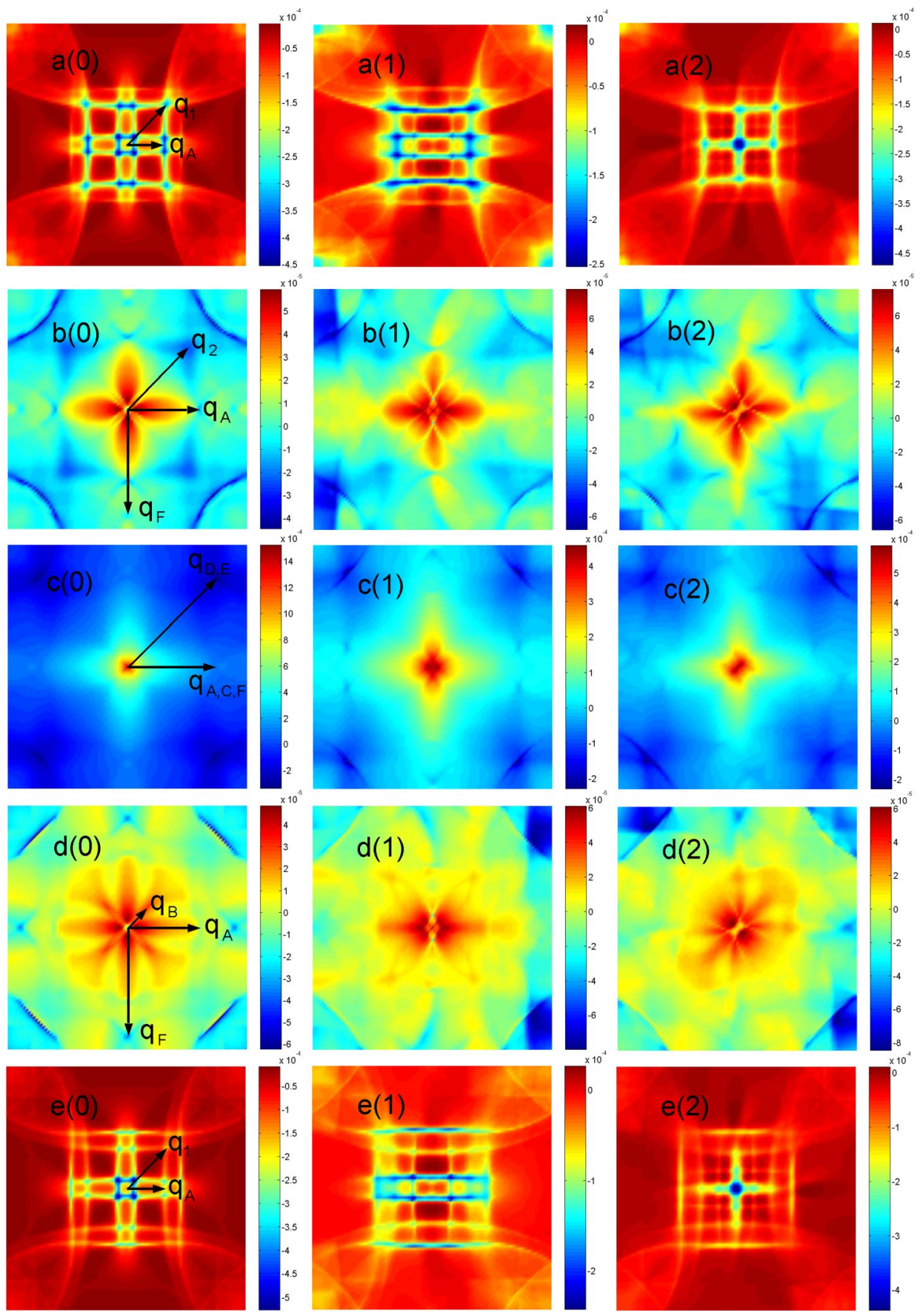

FIG. 4. (Color online) The FCLDOS at $\omega=-44,-16,0,16$, and $44 \mathrm{meV}$ (from top to bottom) and $q=0, q_{c}(0)$, and $q_{c}(\pi / 4)$ (from left to right) in the first Brillouin zone for a unitary defect.

$$
\rho_{\mathrm{expt}}(\mathbf{R}, \omega) \approx \sum_{\delta} \rho(\mathbf{R}+\delta, \omega)
$$

where $\delta$ denotes the nearest neighbor sites of the impurity or $\mathrm{Cu}$ ions.

Taking into account this blocking effect, we present $\rho_{\text {expt }}(\mathbf{R}, \omega)$ curves at the impurity site $(0,0)$ and the points $(0,-1),(1,-1)$, and $(2,0)$ for several $J_{s}$ along $\phi=0$ and $\phi=\pi / 4$, respectively, in Fig. 6. Obviously, the NZBRP on the impurity site and its neighbor sites are strongly suppressed and only slightly broadened with increasing $J_{S}$ in both directions. No splitting is clearly visible. On the other hand, the superconducting coherence peaks shows some sup- pression and splitting near the critical current along the antinodal direction with $\phi=0$, while their separation widens with increasing $J_{s}$ along the nodal direction with $\phi=\pi / 4$. We further notice that the suppression of the NZBRP is insensitive to the direction of $J_{s}$.

A relevant work $^{20}$ studied the NZBRP due to a unitary impurity in the presence of a magnetic field and away from the vortex cores. The magnetic field effect was considered by including the Doppler shifts ${ }^{21}$ in the energies of the quasiparticles and it thus generates nonuniform circulating supercurrent in the sample which is similar but not identical to the case we studied. The NZBRP displayed in Fig. 1 of Ref. 20 is suppressed by the magnetic field (or the supercurrent) 

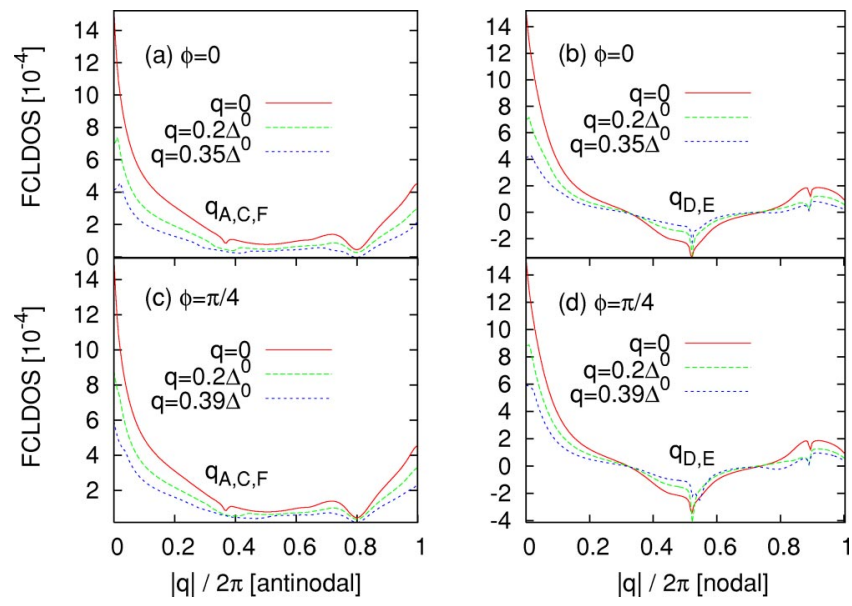

FIG. 5. (Color online) The FCLDOS along the antinodal and nodal directions at $\omega=0 \mathrm{meV}$ and different supercurrents for a unitary defect.

which is like ours, but its width gets narrower as the magnetic field (or $J_{s}$ ) strength becomes larger. This is quite different from what we presented in Fig. 6. The NZBRP displayed in Fig. 1 of Ref. 20 and Fig. 6 in the present paper are both suppressed by the magnetic field B or $J_{s}$, but it appears that their LDOS due to the impurity seems to lose a lot of spectral weight while ours practically remains as a constant as B or $J_{S}$ increases. Further work is needed in order to understand the difference between these two works.

In summary, we have investigated the supercurrent effects on the impurity resonance states in $d$-wave superconductors. The LDOS and FCLDOS patterns induced by a strong impurity have a reflection symmetry if a supercurrent is applied along the antinodal or nodal directions. The suppression and broadening of the resonant peak and the superconducting coherence peaks are due to the anisotropic gap induced by a supercurrent. Future STM experiments need to be performed in order to test these predictions. On the other hand, the (midgap surface state)-induced ZBCP in the tunneling conductance characteristics between a normal metal and a $d$-wave superconductor (dSC) (Ref. 7) looks similar to the strong impurity-induced NZBRP. But their dependences on the applied supercurrent $J_{s}$ are quite different. When both strong defects such as $\mathrm{Cu}$ vacancies and (110)-oriented mi-

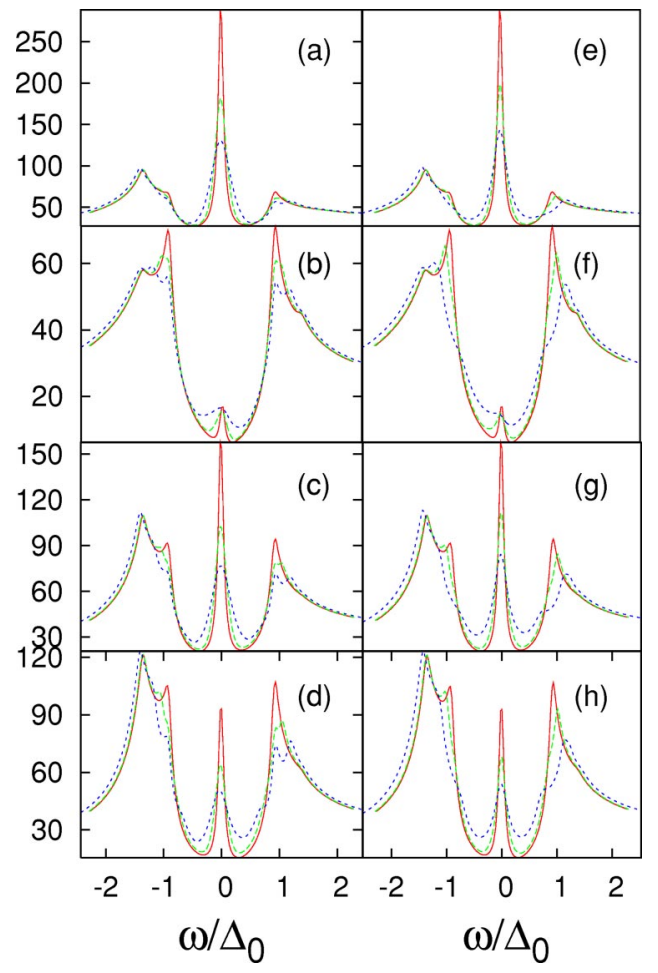

FIG. 6. The predicted, blocking-model-corrected LDOS $\rho_{\text {expt }}(\mathbf{R}, \omega)$ at the sites $(0,0),(0,-1),(-1,-1)$, and $(2,0)$ (from top to bottom) for supercurrents along the antinodal (left) and nodal (right) directions, when a unitary impurity is located at the $(0,0)$ site. Solid: $q=0$; dashed line: $q=0.2 \Delta^{0}$; and dotted line: $q=q_{c}(\phi)$.

crocrystals are present on the surface of an HTS sample, STM experiments should be able to distinguish between these two by analyzing the $J_{s}$ dependences of the zero-bias conductance peak induced by an isolated defect and that induced by the surface midgap states.

The authors wish to thank Professor S. H. Pan for helpful discussions. This work was supported by the Texas Center for Superconductivity and Advanced Materials at the University of Houston and by the Robert A. Welch Foundation (C.S.T.).
${ }^{1}$ C. C. Tsuei and J. R. Kirtley, Rev. Mod. Phys. 72, 969 (2000), and references therein.

${ }^{2}$ C.-R. Hu, Phys. Rev. Lett. 72, 1526 (1994).

${ }^{3}$ A. V. Balatsky, M. I. Salkola, and A. Rosengren, Phys. Rev. B 51, 15547 (1995).

${ }^{4}$ M. I. Salkola, A. V. Balatsky, and D. J. Scalapino, Phys. Rev. Lett. 77, 1841 (1996).

${ }^{5}$ S. H. Pan, E. W. Hudson, K. M. Lang, H. Eisaki, S. Uchida, and J. C. Davis, Nature (London) 403, 746 (2000).

${ }^{6}$ A. M. Zagoskin, Quantum Theory of Many-Body Systems: Techniques and Applications (Springer, New York, 1998).

${ }^{7}$ Degang Zhang, C. S. Ting, and C.-R. Hu, Phys. Rev. B 70,
172508 (2004).

${ }^{8}$ I. Khavkine, H.-K. Kee, and K. Maki, Phys. Rev. B 70, 184521 (2004).

${ }^{9}$ J. Ngai, Y. C. Tseng, P. Morales, V. Pribiag, J. Y. T. Wei, F. Chen, and D. D. Perovic, Appl. Phys. Lett. 84, 1907 (2004).

${ }^{10}$ C. Pepin and P. A. Lee, Phys. Rev. B 63, 054502 (2001).

${ }^{11}$ M. R. Norman, M. Randeria, H. Ding, and J. C. Campuzano, Phys. Rev. B 52, 615 (1994).

${ }^{12}$ J. E. Hoffman, K. McElroy, D.-H. Lee, K. M. Lang, H. Eisaki, S. Uchida, and J. C. Davis, Science 297, 1148 (2002).

${ }^{13}$ K. McElroy, R. W. Simmonds, J. E. Hoffman, D.-H. Lee, J. Orenstein, H. Eisaki, S. Uchida, and J. C. Davis, Nature (London) 
422, 592 (2003).

${ }^{14}$ Qiang-Hua Wang and D.-H. Lee, Phys. Rev. B 67, 020511(R) (2003).

${ }^{15}$ Degang Zhang and C. S. Ting, Phys. Rev. B 67, 100506(R) (2003); Phys. Rev. B 69, 012501(2004).

${ }^{16}$ Lingyin Zhu, W. A. Atkinson, and P. J. Hirschfeld, Phys. Rev. B 69, 060503(R) (2004).

${ }^{17}$ Q. Wang and C.-R. Hu (unpublished).
${ }^{18}$ J.-X. Zhu, C. S. Ting, and C.-R. Hu, Phys. Rev. B 62, 6027 (2000). For an alternative model, see Ref. 19.

${ }^{19}$ I. Martin, A. V. Balatsky, and J. Zaanen, Phys. Rev. Lett. 88, 097003 (2002).

${ }^{20}$ K. V. Samokhin, Phys. Rev. B 68, 104509 (2003).

${ }^{21}$ G. E. Volovik, Pis'ma Zh. Eksp. Teor. Fiz. 58, 457 (1993) [JETP Lett. 68, 469 (1993)]. 